\title{
Keine Pflicht zum bedingungslosen Schwangerschaftsabbruch
}

\author{
Eine Patientin forderte Schmerzensgeld von einer Gynäkologin, \\ weil diese den von der Patientin gewünschten Schwangerschaftsab- \\ bruch wegen ungeklärter Kostenübernahme nicht durchführte.
}

$\mathrm{D}$ ie volljährige Klägerin begab sich für einen Abbruch nach Beratungslösung zur beklagten Gynäkologin, die im Aufklärungsgespräch medizinische Einzelheiten erklärte. Zudem wurde über die finanziellen Voraussetzungen des Eingriffs gesprochen. Der Klägerin wurde freigestellt entweder die Kostendeckungszusage eines Sozialversicherungsträgers mitzubringen oder Barzahlung zu leisten. Die mittellose $\mathrm{Pa}$ tientin wollte ihre Krankenkasse fragen. Aufgrund eines Telefonats ging sie von deren mündlicher Zusage aus, eine schriftliche Zusage erhielt sie aber nie.

In Kenntnis dessen nahm sie dennoch vier Stunden vor dem avisierten Termin ein Zäpfchen zur Weitung des Muttermunds. Die Beklagte lehnte dann aber den Eingriff ab, da eine schriftliche Kostendeckungszusage fehlte und auch keine Barzahlung geleistet wurde. Die Abläufe und Details hierzu in der Praxis stellten sich allerdings höchst strittig dar. Streitig war später zudem, ob das Zäpfchen Beschwerden verursachte. Der Abbruch erfolgte andernorts.

\section{So sah das Gericht den Fall}

Das Amtsgericht Dortmund (Az. 421 C 7915/14) sah letztlich trotz einer äußerst emotionalen Diskussion keinen Grund für Schadensersatzansprüche. Eine Verletzung des Behandlungsvertrages lag nicht vor, da er unter der Bedingung vorausgehender Kostendeckung stand, die von der Klägerin nicht erfüllt wurde. Auch habe zu keiner Zeit die Pflicht bestanden, bedingungslos einen Vertrag zu schließen. Denn nur in Notfällen sei es dem Arzt verwehrt, Behandlungen abzulehnen. Eine solche Lage verneinte das Gericht. Ebenso bestand deliktisch keine Schadensersatzpflicht. Selbst wenn die Patientin aufgrund des Zäpfchens im körperlichen Wohlbefinden beeinträchtigt gewesen sein sollte, seien die Beschwerden nicht Folge einer unmittelbar schuldhaften Handlung der Beklagten.

Auch deren „Unterlassen“ sei nicht zu beanstanden, da sie trotz allem (noch) keine unbedingte Garantenstellung innehatte und auch sonst nicht feststellbar fehlerhaft gehandelt habe. Ein Anspruch aufgrund unterlassener Hilfeleistung ( $\$ 823$ Abs. 2 BGB in Verbindung mit $\$ 323 \mathrm{c}$ StGB) scheiterte am Fehlen eines „Unglücksfalls“, da die Ablehnung des Eingriffs kein „plötzliches unerwartetes Ereignis“, sondern vielmehr für die Klägerin vorhersehbar war.

Ebenso verneint wurde ein Anspruch aufgrund einer Körperverletzung ( $\$ 823$ Abs. 2 BGB in Verbindung mit $\$ 223$ Abs. 1 StGB) durch Verordnung des Zäpfchens. Dessen Gabe erfolgte im Einverständnis der Klägerin, die dies selbst durchführte, obwohl sie wissen musste, dass der Eingriff ohne Kostendeckung nicht erfolgen werde. Auch ein Anspruch wegen Nötigung ( $\$ 823$ Abs. 2 BGB in Verbindung mit $\$ 240$ Abs. 1 StGB) wurde verneint, da nicht zu beanstanden war, dass die Beklagte mit Vorlaufzeit von mehr als einer Woche auf einer Kostendeckung bestand.

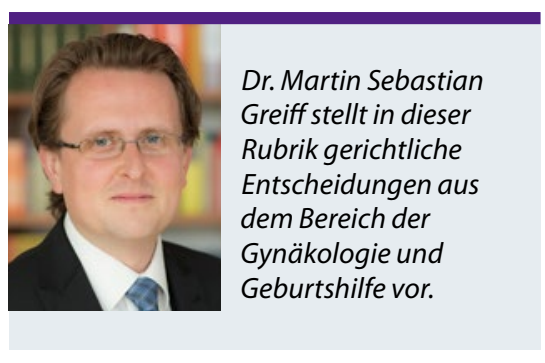

Was bedeutet das Urteil für den klinischen Alltag?

Oft wird vergessen, dass rechtlich abgesehen von Notfällen und speziellen Behandlungssituationen wie im vertragsärztlichen Bereich (vgl. zur Möglichkeit einer Behandlungsablehnung durch den Vertragsarzt nur in begründeten Fällen, $\$ 13$ VII BMV-Ä) kein ärztlicher Zwang besteht, mit Patienten Behandlungsverträge zu schließen; erst recht ist daher auch nicht ohne weiteres bedingungslos ein Schwangerschaftsabbruch durchzuführen. Neben den vom Gericht dazu zivil- und strafrechtlich herausgearbeiteten Aspekten folgt dies berufsrechtlich auch aus $\$ 14$ Abs. 1 Musterberufsordnung. Unangenehm sind solche Auseinandersetzungen dennoch. Nicht selten werden sie nämlich von Strafanzeigen und Beschwerden bei der Ärztekammer begleitet; so auch hier, wobei gleichfalls die Staatsanwaltschaft Dortmund wie die Ärztekammer ihre jeweils zunächst eingeleiteten Ermittlungsverfahren mangels Fehlverhalten wieder einstellten.

\footnotetext{
Dr. Martin Sebastian Greiff, Mag. rer. publ.

Fachanwalt für Medizinrecht Partner der Kanzlei Ratzel Rechtsanwälte Romanstraße 77, 80639 München www.ratzel-rechtsanwälte.de
} 